\title{
HOW DOES THE AMOUNT OF MOVEMENT AND OBSERVER EXPERTISE SHAPE THE PERCEPTION OF MOTION AESTHETICS IN DANCE?
}

original paper

() Wroclaw University of Health and Sport Sciences

DOI: https://doi.org/10.5114/hm.2021.106170

\section{PIA M. VINKEN ${ }^{1}$, THOMAS HEINEN ${ }^{2}$}

${ }^{1}$ Institute of Sport Science, Georg-August-University Göttingen, Göttingen, Germany

${ }^{2}$ Faculty of Sport Science, Leipzig University, Leipzig, Germany

\section{ABSTRACT}

Purpose. Research on empirical aesthetics suggests that specific kinematic parameters are related to the perception of motion aesthetics. Furthermore, an observer's expertise seems to be related to the perception of motion aesthetics when complex biological motion stimuli are present. The central aim of this study was to investigate whether the amount that specific body parts moved during a complex motor skill was related to the perception of motion aesthetics in observers with different levels of sensory-motor expertise.

Methods. Overall, 36 participants divided into 2 groups (18 dancers and 18 non-dancers) were asked to indicate their perceived motion aesthetics when they watched stick-figure video sequences of 3 different semi-standardized dance skills. The stick-figure video sequences were generated from original motion stimuli, and motion aesthetics were measured via Likert scales.

Results. The perception of motion aesthetics in relation to the amount that specific body parts move is skill- and expertisespecific. Dance poses are perceived similarly by dancers and non-dancers, but motion aesthetics during dance jumps and turns are perceived differently. Furthermore, the amount that specific body parts move affects whether the observer perceives the motor skills as more or less aesthetic.

Conclusions. The observer's sensory-motor expertise regarding the observed motor skills can shape their perception of motion aesthetics. The findings of this study demonstrate that there is a skill- and expertise-specific relationship between motion kinematics and motion aesthetics.

Key words: empirical aesthetics, Likert scale, markerless motion capturing, motion perception, stick-figure video sequences

\section{Introduction}

Imagine a dancer performing a complex turn during a dance; the dancer's goal is to execute the turn in an aesthetically pleasing manner. During the creative process, the dancer may implement different dance techniques, motions, and body expressions to satisfy the performance demands and attract the attention of the audience in terms of aesthetics. The resulting impression of the motor skill may differ across the audience members, especially across observers with and without sensory-motor experience related to the observed skill. The following questions arise: What shapes an individual's impression of a skill being performed in terms of aesthetics? Is it affected by the performer's motion kinematics, the observer's exper- tise, or the contextual interaction of both factors? With these questions in mind, the central aim of this study was to investigate whether the amount that specific body parts move during a complex motor skill is related to the perception of motion aesthetics in observers with different experience.

Previous research on (empirical) aesthetics has investigated the properties and features of aesthetic objects, the resulting response mechanisms to such objects by the observer, and the resulting interplay between the object and the observer in a given context [1-5]. When aesthetic objects associated with biological motion are studied, as is typical in artistic sports and performing arts, aspects of an embodiment are additionally taken into account [6-9]. For the purpose and understanding of this paper, the following key

Correspondence address: Pia Maria Vinken, Institute of Sport Science, Georg-August-University Göttingen, Sprangerweg 2, 37075 Göttingen, Germany, e-mail: pia.vinken@sport.uni-goettingen.de

Received: July 1, 2020

Accepted for publication: November 24, 2020

Citation: Vinken PM, Heinen T. How does the amount of movement and observer expertise shape the perception of motion aesthetics in dance? Hum Mov. 2022;23(2):46-55; doi: https://doi.org/10.5114/hm.2021.106170. 
terms are defined: Motion aesthetics is defined as the relationship between properties and features of motion stimuli, the resulting response mechanisms to such stimuli by the observer(s), and the interplay between aspects of the motion stimuli and an observer's response to such stimuli within a given context. Aesthetic motion perception or the perception of motion aesthetics refers to the overall perception of aesthetic (biological) motion stimuli. Furthermore, the term aesthetic features refers to parameters that are related to stimuli's perceived motion aesthetics, which have the potential to increase stimuli's motion aesthetics.

In dance and performing arts, the object of interest can, in general, not be observed independently from the artist's body. Compared with watching nonbiological figural shapes or paintings of landscapes for example, watching human biological movement evokes different response processes by the observer $[3,6,10]$. Although the brain areas whose activity is correlated with aesthetic perception are independent of the stimulus modality [1], previous research on the so-called action observation network [11] has shown that specific brain regions, such as the mirror neuron system, as well as the premotor and occipitotemporal cortices, respond equally to the physical and observational learning of complex skills. Furthermore, it has been argued that cognitive bottom-up mechanisms, as well as relevant top-down mechanisms, are integrated during the visual perception of biological motion [10]. Consequently, to shed light on the interplay of aesthetic motion perception, the relationship between the objectdriven aesthetic features of motion stimuli and an observer expertise to such motion stimuli should be investigated cohesively. In this study, contextual factors were controlled for and original motion stimuli were reduced with the use of stick-figure video sequences, thereby preventing biases related to visual perception [10].

The perception of motion aesthetics in complex motor skills seems to be expertise-specific. The findings of studies among performers with different levels of expertise are quite consistent. Motor skills presented by performers with higher expertise levels are perceived as more aesthetically pleasing than the same motor skills exhibited by performers with lower expertise levels [12, 13]. However, the relationship between the observer's sensory-motor and contextual experience and the perception of motion aesthetics seems to be complex.

Expert and novice observers of dance seem to implement different strategies when watching [14], discriminating [15], and evaluating [16] dance sequences.
Novice dance observers, compared with expert dance observers, have longer fixation times and different fixation locations when watching a dance sequence [14]. The observer's expertise regarding the perceived motion stimuli may thus be related to what the observer can perceive when watching a motor skill, which then may also be related to their aesthetic motion perception (cf. aesthetic triad) [2]. For example, female dancers are more able to discriminate point-light displays of female dance skills than male dancers. Although dancers in general outperform both male and female non-dancers, authors suggest that female dancers outperform their male counterparts as well as female and male non-dancers because they have both visual and motor experience of the female dance skills that are discriminated [15]. Furthermore, naïve dance observers seem to aesthetically prefer the dance skills that they do not think they are able to perform [16]. Consequently, the degree of (multi)sensory experience seems to be related to an observer's ability to perceive a motor skill's motion aesthetics. Whether observers with sensory-motor experience differ in their perception of motion aesthetics compared with observers without sensory-motor experience watching the same motor skills is still open for investigation, and the answer to this question may shed light on whether motion aesthetics are more strongly related to the object, the performer, or the interaction of both factors.

Previous research on motion aesthetics has pointed out specific object-driven aesthetic features. On the one hand, qualitative and dance-specific motion descriptors, such as the direction of movement, a high (joint) flexibility of the performer, or a large and diagonal spread of the body during postures, have been identified $[6,7,17]$. On the other hand, quantitative and motion-related kinematic parameters, such as the amplitude or range of motion of specific joints, balance time, vertical angles of body segments, jump height, (turning) speed and movement velocity, as well as motion smoothness [6, 12, 18, 19], have been described. Although methodological differences within these studies impede the identification of overarching aesthetic features, the relationship between kinematic parameters and the perception of motion aesthetics has been shown to be skill-dependent. The perceptual ratings of dance skills seem specific to the requirements and mechanics of the motor skill $[6,18]$. If a motor skill requires the performer to jump, kinematic parameters of jumping ability seem to be related to the aesthetic perception of the skill. In contrast, if as kill requires balance and limb displacement, the balance time and elongation angle of the moving limb may be 
P.M. Vinken, T. Heinen, Perception of motion aesthetics in dance skills

the kinematic parameters related to the observer's aesthetic ratings $[17,18]$. Therefore, the relationship between motion kinematics and motion aesthetics is quite skill-specific, and skill-independent relationships between motion kinematics and motion aesthetics are limited. It is thus assumed that comparing stickfigure video sequences of motor skills with different requirements and modifying a skill-independent kinematic parameter can provide information to fill the research gap and is a useful approach, especially to investigate the relationship between object-driven kinematic parameters, such as the amount that specific body parts move, and the perception of motion aesthetics.

In general, the human visual system allows an individual to perceive moving lines, corresponding body angles, and motions in space when they observe stickfigure video sequences [10]. Regarding angle perception and discrimination, psychophysical theories have suggested critical values for just-noticeable differences (JNDs) in visual perception (cf. Weber's law or theory of line combinations) [20]. In psychophysical research, JNDs between $1.5^{\circ}$ and $3.5^{\circ}$ indicate that the human visual system detects angular changes of at least approximately $3^{\circ}$. Those findings are hardly transferrable to the perception of complex biological motor skills, where multiple body angles and segment orientations are holistically viewed and perceived. However, research on body angle discrimination and related visual biases has suggested that there is a critical JND in the magnitude of body angles that can be perceived. Body angles above a conservative perceptual threshold of $15^{\circ}$ per body segment seem to be applicable for complex biological motion kinematics [20-23]. For example, Giblin et al. [21] investigated whether expert coaches, novice coaches, and tennis players differred in their ability to detect kinematic changes in tennis serves. The angle of maximum knee flexion, the angle of maximum trunk rotation, and the height of ball toss were manipulated by a tennis player executing the tennis serve. Observers wrote down whether they perceived changes in point-light sequences of the manipulated tennis serves. The results indicate that coaching expertise provides no additional benefits in detecting holistic kinematic changes. However, with regard to perceived changes in knee flexion, experts seem more sensitive in their ability to detect knee flexion angle differences between $-5.8^{\circ}$ and $+7.5^{\circ}$ in a reference tennis serve [21].

Investigating whether the amount that specific body parts move affects the perceived aesthetics of dance skills requires a skill-independent comparison of the motion kinematics with the perceived motion aes- thetics. Measuring the amount of movement in complex motor skills such as dance jumps, poses, and turns by calculating and comparing the summed angular motion difference per second of movement enables kinematic parameters to be compared across different skills. Furthermore, this skill-independent approach and the kinematic measure of the summed angular motion difference per second of movement may help reveal previous research investigating the relationship between motion kinematics and motion aesthetics quantitively in terms of skill-specific kinematic parameters [12, 18, 19], qualitatively [13, 24], and technically [24].

In sum, previous research has suggested that there are specific kinematic parameters related to the perception of motion aesthetics. However, the formerly assessed kinematic parameters seem to be strongly skill-specific, and skill-independent parameters and their relation to motion aesthetics still need to be investigated. Furthermore, an observer's expertise seems to be related to the perception of motion aesthetics and should be taken into account when one aims to identify fundamental kinematic parameters of motion aesthetics. According to previous research, observers' holistic impression when aesthetically perceiving complex motor skills has not yet been studied quantitively or skill-independently [17, 18, 25]. Additionally, observers with different expertise may implement different perceptual strategies when observing motion stimuli, which then shape their motion aesthetics differently [13, 14]. Consequently, measuring kinematic variations of complex motor skills in combination with subjective indications of motion aesthetics from observers with different sensory-motor expertise to such motor skills seems to be an appropriate approach to obtain insight into the relation between motion kinematics and motion aesthetics. Thus, it seems to be the method in which observers can indicate their perceived motion aesthetics in the most unbiased manner possible [3]. Furthermore, implementing rating scales, such as Likert scales, is appropriate when large numbers of displays have to be rated aesthetically [26].

In this study, it was hypothesized that the amount that specific body parts move during a complex motor skill would be related to the perception of motion aesthetics by observers with different experience. More precisely, it was hypothesized that the amount that specific body parts move and its relation to the perception of motion aesthetics would differ depending on the dance skill being performed. Second, it was hypothesized that the perception of motion aesthetics by observers with and without sensory-motor experi- 
ence would differ depending on the amount that specific body parts move. Moreover, how the amount that specific body parts move shapes the perception of dance skills regarding the extent to which they are considered aesthetically was explored.

\section{Material and methods}

\section{Participants}

The study sample consisted of 36 participants in 2 groups: $n_{1}=18$ participants with dance experience in classical, modern, and jazz dance (dancers) and $n_{2}=$ 18 participants without dance experience (non-dancers). The dancers (16 females, 2 males) were $29 \pm 11$ years old and had an average of $16 \pm 12$ years of dancing experience with $6 \pm 5$ training hours per week. The non-dancers (9 females, 9 males) were $22 \pm 2$ years old and reported not having any dance experience but participated in different sports regularly with an average of $6 \pm 5$ years of general sport experience and $3 \pm 2$ hours of practice per week. The subjects' task was to indicate their perceived motion aesthetics when they watched stick-figure video sequences of 3 different semi-standardized dance skills, namely, jumps, poses, and turns.

An additional stimulus group of 9 experienced female dancers (mean age: $29 \pm 3$ years) was recruited to generate the video stimuli. The dancers reported having substantial experience in different dance styles, such as classical dance, modern dance, and jazz dance. Their average duration of dancing experience was $21 \pm$ 8 years, with $4 \pm 1$ hours per week of regular practice. The dancers' task was to perform semi-standardized jumps, poses, and turns.

The study participation was voluntary. All participants received an hourly credit if administrable for their studies.

\section{Instruments}

\section{Stimuli generation}

The procedure used to generate stimuli was similar to the one proposed by Vinken and Heinen [17]. For the sake of clarity, the relevant steps are described here.

The dancers who visited the gymnasium were informed about the general purpose of the study, as well as the video stimuli generation process. Each dancer filled out a short questionnaire about their dance experience. The dancers were asked to individually warm up and prepare for the upcoming task. Video stimuli for the 3 dance skills, namely, jumps, poses, and turns, were generated randomly for each dancer.

Each dancer was informed about the motion prerequisites of the dance skill being performed first, for example, the jump. The instructions and prerequisites of the 3 dance skills were as follows. For the dance jump, the dancer was told: 'Stand upright with your feet hip-width apart and your arms hanging loosely. Jump from the left leg with a $45^{\circ}$ turn to land on the right leg and then come back to the upright stance with your feet hip-width apart and arms positioned to the side of the body. Please show variations of this jump by individually varying the movement of the legs, arms, trunk, and whole body as well as accentuation and complexity'. For the dance pose, the dancer was told: 'Stand upright with your feet hip-width apart and arms hanging loosely. Use the left leg as the standing leg, exhibit a one-legged pose and then come back to the upright stance with your feet hip-width apart and arms positioned to the side of the body. Please show variations of this pose by individually varying the movement of the legs, arms, trunk, and whole body as well as accentuation and complexity'. For the dance turn, the dancer was told: 'Stand upright with your feet hip-width apart and arms hanging loosely. Do a $450^{\circ}$ turn to the left with your right leg as the standing leg and then come back to the upright stance with your feet hip-width apart and arms positioned to the side of the body'.

The dancer was allowed to individually practise the skill and ask questions about the movement variations, instructions, and the video stimuli generation process. Then, each dancer was asked to perform at least 4 variations of the first dance skill. Afterward, each dancer was asked whether they were satisfied with their performance or wanted to do another repetition or variation. When at least 4 variations of the first dance skill were successfully performed and captured, the dance skill performance and video capture processes were repeated twice for the remaining dance skills. When each dancer performed at least 4 variations of each dance skill, she was debriefed.

The dance skills were performed in a capture area of $5 \times 5$ meters, and they were videotaped by 6 video cameras sampling at $60 \mathrm{~Hz}(640 \times 480$ pixels $)$. Each recorded video sequence was processed with a silhouette-based computer-based algorithm to extract the movement kinematics (iPi Motion Capture ${ }^{\mathrm{TM}}$, iPi Soft, Russia). The video recordings from all 6 cameras were used to generate a 3D volume model of a human body consisting of a head, a trunk, 2 upper and lower limbs, 
P.M. Vinken, T. Heinen, Perception of motion aesthetics in dance skills

2 hands, 2 thighs and shanks, 2 feet, and the appropriate joints, namely, the neck, shoulders, elbows and wrists, chest, hips, knees, and ankles.

Stick-figure video sequences were generated from the extracted movement kinematics. To that end, the original video recordings were reduced to the kinematic motion information, which allowed potential contextual and bodily biases to be controlled [10]. To achieve a sufficient variety of stimuli dance skills, uniform variations of dance skills were grouped, and one stick-figure sequence was randomly selected for the stimuli sample. The uniform variations consisted, for example, of equal implementation of arm and leg displacement, head and trunk involvement, and dance skill-specific movement vocabulary. Ten stickfigure video sequences of each dance skill were selected for stimuli presentation and evaluation, thus representing a sufficient variety of different jumps, poses, and turns. At the end of the steps mentioned above, there were 30 stick-figure video sequences of dance skills: 10 for jumps, 10 for poses, and 10 for turns.

\section{Stimuli evaluation}

The stick-figure video sequences of the dance skills were prepared for a randomized stimuli presentation. Each video sequence of the first dance skill, for example, the pose, was presented twice at the original tempo on a $2.5 \times 1.8$-meter projection screen. The dancers and the non-dancers were asked to respond to the question: 'How do you perceive the sequence aesthetically?' [16, 27] by selecting the corresponding number on a 7-point Likert scale ranging from -3 to +3 [28] on a piece of paper. When the first dance skill was evaluated, the same procedure was repeated for the remaining 2 dance skills. This procedure took approximately 30 minutes per participant. Neither the terms 'aesthetics' nor 'aesthetically' were defined so that the participants answered the question in the least unbiased manner possible [3]. Ten responses were recorded per participant and dance skill, which resulted in a total of 1080 values that were used for data analysis.

After the participants' responses, the dance skills were divided into 2 groups, the 5 most and 5 least aesthetically perceived ones. Therefore, the median splits of the participants' responses to the dance skills were assessed for each dance skill [28].

\section{Procedure}

The dance skills were evaluated in 3 phases. First, each dancer and non-dancer was invited separately to a laboratory room at the local university. They were informed about the general purpose of this study, signed an informed consent form, and completed a short questionnaire about their dance experience. Afterward and before the data were collected, the experimenter introduced the evaluation procedure by showing exemplary stick-figure video sequences and explaining the data collection process. Likert scale assessments were performed separately for each of the 3 dance skills, and the jumps, poses, and turns were presented in a random order for the participants.

Second, to collect the study data, 10 stick-figure video sequences of the first dance skill, for example, the turn, were randomly presented to each participant at the original tempo. Each stick-figure video sequence was presented twice in the middle of the screen. After each turn, the participant responded to the question: 'How do you perceive the sequence aesthetically?,' which was written on the presentation slide. The responses were recorded on a piece of paper with a 7-point Likert scale. After the participant responded, the stickfigure sequence for the next turn was presented. This procedure was repeated for each of the 10 turns. After evaluating the first dance skill, the participants were allowed to take a short break. The same procedure was repeated twice for the remaining dance skills. The participants were not pressured to respond quickly, but they were instructed to indicate the first response that came to mind.

Third, after data collection, each participant was debriefed and received an hourly credit if administrable for their studies.

\section{Data analysis}

Data analysis was conducted with the statistical software R (R Core Team, 2018). The data set of one trial contained angle-time information for 63 variables that were divided into 3 categories: (1) wholebody translation was defined by 3 variables ( $\mathrm{x}-$, $\mathrm{y}^{-}$, and z-position of body); (2) whole-body rotation was defined by another 3 variables ( $\mathrm{x}-, \mathrm{y}-$, and $\mathrm{z}$-rotation of body); (3) the joint movements of each of the 19 joints were defined by 3 variables ( $\mathrm{x}-, \mathrm{y}-$, and $\mathrm{z}$-rotation of each joint). The trials lasted an average of 4.39 seconds with a standard deviation of \pm 0.57 seconds. First, each trial's variables were time-normalized by spline interpolation to ensure structural comparability between trials of different durations [29]. Second, the time-normalized trials of each dance skill in each of the 2 categories (more vs. less aesthetic) were averaged (see Stimuli evaluation). This approach resulted 
in one time-normalized data frame representing an 'average' performance of a 'more aesthetic' skill and one time-normalized data frame representing an 'average' performance of a 'less aesthetic' skill. This was done for each of the 3 skills. Third, the summed angular motion differences of the variables corresponding to each body segment (1. movements of the left arm, 2. movements of the right arm, 3. movements of the left leg, 4. movements of the right leg, and 5. movements of the trunk and head) were calculated for each of the 3 skills to assess the similarity between more and less aesthetic performances [30].

The summed angular motion differences of the body segments were normalized to skill duration so that between-trial comparisons could be performed. This calculation allowed the extent to which each variable category contributed to the overall impression of motion aesthetics to be estimated. A positive difference in one variable category thus indicates that this category shows a larger amount of movement for more aesthetic skills. In contrast, a negative difference implies that this category shows a larger amount of movement for less aesthetic skills. A summed difference of zero denoted that both the more and less aesthetic skills exhibited the same amount of movement in the particular categories. For example, a movement of $90^{\circ}$ in the elbow joint in one second of movement and another movement of $45^{\circ}$ in the elbow joint within the same second were represented as a summed angular motion difference of $45^{\circ}$. This procedure was performed to compare the dancers' and non-dancers' indications of perceived motion aesthetics by the level of the observer's expertise.

Finally, a conservative critical value of $15^{\circ}$ was chosen to differentiate between a perceptually relevant amount of movement, assuming that perceptual salience was larger for the larger summed angular motion differences [20-23].

\section{Ethical approval}

The research related to human use has complied with all the relevant national regulations and institutional policies, has followed the tenets of the Declaration of Helsinki, and has been approved by the authors' institutional ethics committee.

\section{Informed consent}

Informed consent has been obtained from all individuals included in this study.

\section{Results}

In accordance with the median split procedure for the more vs. less aesthetic dance skills, a descriptive inspection of the means showed a clear distinction in the Likert scale ratings of more and less aesthetic dance skills among the 3 dance skills and the 2 observer groups (dancers and non-dancers). For the dancers, the rating was $1.21 \pm 0.35$ vs. $-0.20 \pm 0.78, t(8)=3.71$, $p<0.05$ for the jumps, $1.15 \pm 0.63$ vs. $-0.39 \pm 0.58$, $t(8)=4.54, p<0.05$ for the poses, and $1.08 \pm 0.34$ vs. $-0.62 \pm 0.82, t(8)=4.10, p<0.05$ for the turns. For the non-dancers, it was $1.64 \pm 0.63$ vs. $-0.40 \pm 1.03$, $t(8)=4.03, p<0.05$ for the jumps, $1.33 \pm 0.71$ vs. $-0.29 \pm 0.42, t(8)=4.51, p<0.05$ for the poses, and $1.59 \pm 0.50$ vs. $-0.34 \pm 0.81, t(8)=4.70, p<0.05$ for the turns. On average, the rating scales of more vs. less aesthetic dance skills differed by 1.7 points, equalling $24 \%$ of the overall scale and a range of 1.41-2.04.

Figure 1 shows the dancers' and non-dancers' perceived motion aesthetics of the dance skills and their summed angular motion differences (means and $95 \%$ confidence intervals). A summed angular motion difference of $\pm 15^{\circ}$ represents a conservative critical value [20-23]. Values above $+15^{\circ}$ correspond to the dance skills showing a larger amount of movement in the corresponding body part which is perceived as more aesthetic. Values below $-15^{\circ}$ correspond to the dance skills showing a larger amount of movement in the corresponding body part which is perceived as less aesthetic.

Both dancers and non-dancers perceived poses with a larger amount of movement in the right arm and the left standing leg as less aesthetic. Dancers' and non-dancers' perceptions of motion aesthetics differed for jumps and turns. Concerning dance jumps, dancers perceived dance jumps with a larger amount of movement in the left arm as more aesthetic and dance jumps with a larger amount of movement in the trunk and head as less aesthetic. Non-dancers perceived dance jumps with a larger amount of movement in the left take-off leg as less aesthetic. Concerning turns, dancers perceived turns with a larger amount of movement in the left gesture leg as more aesthetic, while non-dancers perceived turns with a larger amount of movement in the left arm as more aesthetic.

\section{Discussion}

The central aim of this study was to investigate whether the amount that specific body parts move dur- 


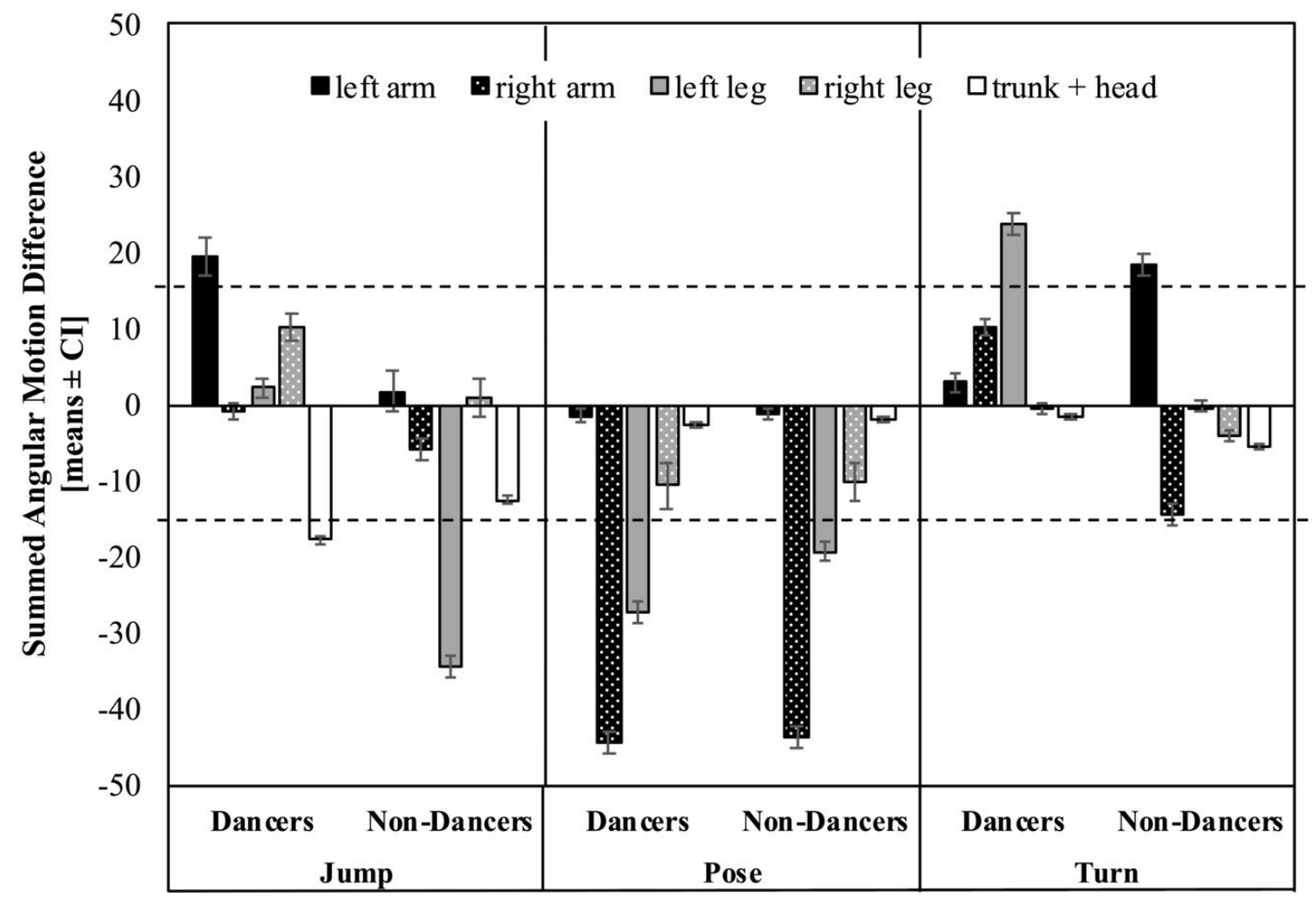

Figure 1. Illustration of the dancers' and non-dancers' perception of motion aesthetics of the dance skills in relation to the summed angular motion differences in the corresponding body parts (means and 95\% confidence intervals [CI]). The dashed lines indicate a conservative critical value. Values above $+15^{\circ}$ correspond to the dance skills showing larger summed angular motion differences in the corresponding body part which are perceived as more aesthetic. Values below $-15^{\circ}$ correspond to the dance skills showing larger summed angular motion differences in the corresponding body part

which are perceived as less aesthetic. The summed angular motion differences are normalized to skill duration,

thus representing the amount of movement in the corresponding body part per second of movement

ing a complex motor skill was related to the perception of motion aesthetics by observers with different experience. It was hypothesized that the amount that specific body parts moved and its relation to the perception of motion aesthetics would differ depending on the dance skill and the observer's sensory-motor expertise. Additionally, it was explored how the amount that specific body parts moved shaped the perception of dance skills, which are perceived as more or less aesthetic. The results indicate that the perception of motion aesthetics in relation to the amount that specific body parts move is skill- and expertise-specific. For dance poses, the amount that specific body parts move affects dancers' and non-dancers' perception of motion aesthetics similarly, whereas dancers' and non-dancers' perceptions of motion aesthetics differ for dance jumps and turns.

Interestingly, for dance poses, a larger amount of movement in the right arm and the left standing leg was perceived as less aesthetic by both dancers and non-dancers. This finding may indicate that, concerning dance poses, less is more. Observers with and with- out sensory-motor experience related to the observed stimuli may perceive several accentuations within the motor skill as less aesthetic than a single accentuation [17]. Furthermore, this finding implies that simple dance skills are perceived as more aesthetic when they are executed softly and expansively [25]. However, in this case, simplicity is not related to the difficulty of the executed motor skill but rather the overall amount that the body segments move. In general, dance poses may demonstrate the performer's ability to remain balanced while reducing the magnitude of movement of specific body parts. Therefore, observers seem to prefer motor skills that may focus, for example, on a single but large inclination of the gesture leg compared with several accentuations where the gesture leg is, for example, moved up, then down, and additionally rotated at the hip and knee joint.

In dance jumps, dancers' and non-dancers' perceptions of motion aesthetics differed concerning the amount that specific body parts moved. Dancers perceived a larger amount of movement in the left arm as more aesthetic and a larger amount of movement in 
the head and trunk as less aesthetic. In contrast, nondancers perceived a larger amount of movement in the left take-off leg as less aesthetic. A larger amount of movement in the head and trunk may be related to the performer's inability to stabilize the head and trunk while implementing arms and legs to perform the dance jump. To master a jump skill, the performer must be able to take off from the floor, remain a sufficient flight phase, and then prepare an appropriate landing. In general, a performer's aim during jumping is to achieve the impression of ease [6]. A lack of strength and motor control for explosive jumping can be partly compensated by using a countermovement in the trunk during take-off and landing [28]. This aspect may be 'detectable' or not depending on the observers' sensory-motor and contextual experience related to the observed skill and thus may be related to their perception of motion aesthetics.

Previous research has indicated that motor skills involving energetic jumping are perceived as more aesthetic than motor skills lacking significant horizontal or vertical displacement of the performer in space [6]. Moreover, the extent to which limbs move during jumping seems to be related to the observer's aesthetic perception of a jump. When specific body parts, namely, the left arm, move by a larger amount, dancers with sensory-motor experience related to the observed skill may perceive the movement to be more aesthetic. However, when different body parts, namely, the left take-off leg, move by a larger amount, observers without sensory-motor experience related to the observed skill may perceive the movement to be less aesthetic. Therefore, the level of accentuation and implementation of the moving limbs during jumping, as well as the observer's expertise level, can affect the perception of motion aesthetics in general.

Regarding turns in dance, the dancers' and nondancers' perceptions of motion aesthetics differed in relation to the amount that the left gesture leg and the left arm moved. Dancers perceived dance turns in which the left gesture leg moved more as more aesthetic. Non-dancers perceived dance turns in which the left arm moved more as more aesthetic. In general, moving the limbs away from the performer's longitudinal axis increases the performer's moment of inertia, which challenges physical laws [29] and thus the performer's ability to successfully master turning. Hence, this study results suggest that motor skills which seem more difficult and challenging to perform are perceived as more aesthetic [16].

Dancers and non-dancers perceive motion aesthetics differently depending on the amount that different body parts move, which may indicate that observers with and without sensory-motor expertise may implement different strategies when perceiving and evaluating motion aesthetics. As eye-tracking studies suggest, expert and novice dance observers fixate on different body parts when watching a dance performance [14]. Therefore, the amount that different body parts move may be related to dancers' and non-dancers' perception of motion aesthetics. However, according to the results of this study, non-dancers perceive dance turns in which the left or the right arm moves a large amount differently in relation to motion aesthetics. Non-dancers perceive dance turns with a large amount of movement in the right arm as less aesthetic, while dance turns with a large amount of movement in the left arm are perceived as more aesthetic. This finding may be related to the aesthetic fundamental of symmetry $[1,26]$. The dance turn performed in this study used the right leg as the standing leg. One may argue that non-dancers seek symmetry in the limbs of the same body side. While the right standing leg shows a restricted amount of movement, non-dancers may perceive asymmetry when the corresponding right arm shows a large amount of movement, an aspect that may shape motion aesthetics in such a way that the corresponding dance turns are perceived as less aesthetic.

The findings of this study reveal a relationship between motion kinematics and motion aesthetics. The summed angular motion difference and amount that specific body parts move are parameters that allow the results to be interpreted independently of the skill. It is assumed that in the perception of complex motor skills, motion aesthetics and motion kinematics are related and that, specifically, motion kinematics affect motion aesthetics [13, 19]. Different skills can be compared via a single kinematic parameter. This finding expands upon previous knowledge on the relationship of skill-specific parameters and their relations to the perception of motion aesthetics. Finally, the expertisespecific relationship between motion kinematics and motion aesthetics builds upon previous research among observers with $[12,17]$ or without $[6,12,16,19]$ sensory-motor experience. Therefore, future studies should replicate these methods across different groups of observers. It seems that the relationship between motion kinematics and motion aesthetics is expertise-specific [13-15] and embodied [7-9].

When interpreting the results of this study, the following limitations should be taken into account. First, the summed angular motion difference, representing the amount that specific body parts moved, was considered a kinematic parameter. It remains 
unclear whether focusing on a single joint instead of specific body parts can deepen our understanding of underlying motion kinematics. However, concentrating on the amount that specific body parts move by summing the kinematics of several body segments seems to provide a holistic assessment of movements compared with, for example, the inspection of single body joints and the amount that they move. Second, behavioural measures were implemented when observers were asked to indicate their perception of motion aesthetics after observing stick-figure video sequences of original dance skills. Future studies should additionally incorporate other measures, such as neurological or eye-tracking measures, to determine whether observers with different sensory-motor experience implement different strategies when assessing the motion aesthetics of complex motor skills. Third, the original motion stimuli were reduced to stick-figure video sequences. Future studies that manipulate a standardized motor skill by, for example, artificially manipulating the kinematic parameters, may deepen our understanding of the relationship between motion kinematics and motion aesthetics.

From the results of this study, the following practical implications can be derived. When aiming to create and perform aesthetic motor skills, the sensorymotor and contextual expertise of the observer and target audience should be taken into account. Depending on the observed skill, observers with sensory-motor experience perceive dance skills in which specific body parts move more as more or less aesthetically pleasing than observers without sensory-motor expertise. Furthermore, the amount that specific body parts move and its relation to the perception of motion aesthetics are skill-specific. While dancers and non-dancers similarly perceive dance poses in which the right arm and left standing leg move a large amount as less aesthetic, dancers' and non-dancers' perceptions of motion aesthetics differ for dance jumps and turns. For example, dance turns with a large amount of movement in the left gesture leg are perceived as more aesthetic by dancers, whereas non-dancers perceive dance turns with a large amount of movement in the left arm as more aesthetic.

Finally, performers, coaches, choreographers, and researchers should keep in mind that the amount that specific body parts move when a motor skill is being performed may shape the perception of motion aesthetics, especially among observers with different sensory-motor expertise related to the observed motor skill. Therefore, ways of optimizing dance motions to make them able to trigger the observer's aesthetic per- ception should be developed [7]. As a result, fruitful interdisciplinary collaboration between researchers and dancers may develop for the study of motion aesthetics.

\section{Conclusions}

The perception of motion aesthetics arises from a complex interplay between aspects of the object, the observer, and the context. The findings of this study demonstrate that there is a skill- and expertise-specific relationship between motion kinematics and motion aesthetics. It is concluded that an observer's sensory-motor and contextual expertise related to the observed motor skills can shape the perception of motion aesthetics. In other words, the amount that specific body parts move is related to an observer's perception of motion aesthetics. However, observers with different levels of expertise partly perceive different motion kinematics as more or less aesthetically pleasing. Although original motion stimuli were reduced to stick-figure video sequences of dance skills, it is assumed that this study results are generalizable to original video sequences, laboratory performances, and original performances. Fruitful collaboration between researchers and dancers regarding the study of motion aesthetics may develop in the future, broadening our knowledge on the relationship between motion kinematics and motion aesthetics. When researchers understand the underlying processes of motion aesthetics, practitioners will be able to develop and justify their (implicit) knowledge of creating and performing aesthetic motion stimuli.

\section{Acknowledgements}

We acknowledge the support by the Open Access Publication Funds of the Göttingen University and thank Damian Jeraj and Lisa Riedel for their comments and support concerning a previous version of this manuscript.

\section{Disclosure statement}

No author has any financial interest or received any financial benefit from this research.

\section{Conflict of interest}

The authors state no conflict of interest.

\section{References}

1. Brielmann AA, Pelli DG. Aesthetics. Curr Biol. 2018; 28(16):R859-R863; doi: 10.1016/j.cub.2018.06.004. 
2. Chatterjee A, Vartanian O. Neuroaesthetics. Trends Cogn Sci. 2014;18(7):370-375; doi: 10.1016/j.tics.2014.03. 003.

3. Jacobsen T. Bridging the arts and sciences. A framework for the psychology of aesthetics. Leonardo. 2006; 39(2):155-162; doi: 10.1162/leon.2006.39.2.155.

4. Leder H, Nadal M. Ten years of a model of aesthetic appreciation and aesthetic judgments: the aesthetic episode - developments and challenges in empirical aesthetics. Br J Psychol. 2014;105(4):443-464; doi: 10.1111/bjop.12084.

5. Pearce MT, Zaidel DW, Vartanian O, Skov M, Leder H, Chatterjee A, et al. Neuroaesthetics: the cognitive neuroscience of aesthetic experience. Perspect Psychol Sci. 2016;11(2):265-279; doi: 10.1177/174569161562 1274.

6. Calvo-Merino B, Jola C, Glaser DE, Haggard P. Towards a sensorimotor aesthetics of performing art. Conscious Cogn. 2008;17(3):911-922; doi: 10.1016/j.concog.2007. 11.003.

7. Christensen JF, Calvo-Merino B. Dance as a subject for empirical aesthetics. Psychol Aesthet Creat Arts. 2013;7(1):76-88; doi: 10.1037/a0031827.

8. Kirsch LP, Urgesi C, Cross ES. Shaping and reshaping the aesthetic brain: emerging perspectives on the neurobiology of embodied aesthetics. Neurosci Biobehav Rev. 2016;62:56-68; doi: 10.1016/j.neubiorev.2015.12. 005.

9. Montero B. Practice makes perfect: the effect of dance training on the aesthetic judge. Phenom Cogn Sci. 2012; 11:59-68; doi: 10.1007/s11097-011-9236-9.

10. Blake R, Shiffrar M. Perception of human motion. Annu Rev Psychol. 2007;58:47-73; doi: 10.1146/annurev. psych.57.102904.190152.

11. Cross ES, Kraemer DJM, de C Hamilton AF, Kelley WM, Grafton ST. Sensitivity of the action observation network to physical and observational learning. Cereb Cortex. 2009;19(2):315-326; doi: 10.1093/cercor/bhn083.

12. Bronner S, Shippen J. Biomechanical metrics of aesthetic perception in dance. Exp Brain Res. 2015;233(12): 3565-3581; doi: 10.1007/s00221-015-4424-4.

13. Zamparo P, Carrara S, Cesari P. Movement evaluation of front crawl swimming: technical skill versus aesthetic quality. PLoS One. 2017;12(9):e0184171; doi: 10.1371/journal.pone.0184171.

14. Stevens C, Winskel H, Howell C, Vidal L-M, Latimer C, Milne-Home J. Perceiving dance: schematic expectations guide experts' scanning of a contemporary dance film. J Dance Med Sci. 2010;14(1):19-25.

15. Calvo-Merino B, Ehrenberg S, Leung D, Haggard P. Experts see it all: configural effects in action observation. Psychol Res. 2010;74(4):400-406; doi: 10.1007/ s00426-009-0262-y.

16. Cross ES, Kirsch L, Ticini LF, Schütz-Bosbach S. The impact of aesthetic evaluation and physical ability on dance perception. Front Hum Neurosci. 2011;5:102; doi: 10.3389/fnhum.2011.00102.
17. Vinken PM, Heinen T. Perceived aesthetic features differentiating between complex artistic dance skills of varying style. Sci Gymnast J. 2020;12(2):119-133.

18. Chang M, Halaki M, Adams R, Cobley S, Lee K-Y, O'Dwyer N. An exploration of the perception of dance and its relation to biomechanical motion: a systematic review and narrative synthesis. J Dance Med Sci. 2016; 20(3):127-136; doi: 10.12678/1089-313X.20.3.127.

19. Torrents C, Castañer M, Jofre T, Morey G, Reverter F. Kinematic parameters that influence the aesthetic perception of beauty in contemporary dance. Perception. 2013;42(4):447-458; doi: 10.1068/p7117.

20. Xu Z-X, Chen Y, Kuai S-G. The human visual system estimates angle features in an internal reference frame: a computational and psychophysical study. J Vis. 2018; 18(13):10; doi: 10.1167/18.13.10.

21. Giblin G, Farrow D, Reid M, Ball K, Abernethy B. Does perceptual or motor experience influence the perception of global and joint-specific kinematic changes in complex movement patterns? Atten Percept Psychophys. 2016;78:1781-1793; doi: 10.3758/s13414-0161167-7.

22. Plessner H, Schallies E. Judging the cross on rings: a matter of achieving shape constancy. Appl Cogn Psychol. 2005;19(9):1145-1156; doi: 10.1002/acp.1136.

23. Weir PL, Holmes AM, Andrews DM, Albert WJ, Azar NR, Callaghan JP. Determination of the just noticeable difference (JND) in trunk posture perception. Theor Issues Ergon Sci. 2007;8(3):185-199; doi: 10.1080/ 14639220500232446.

24. Christensen JF, Pollick FE, Lambrechts A, Gomila A. Affective responses to dance. Acta Psychol. 2016;168: 91-105; doi: 10.1016/j.actpsy.2016.03.008.

25. Christensen JF, Nadal M, Cela-Conde CJ, Gomila A. A norming study and library of 203 dance movements. Perception. 2014;43(2-3):178-206; doi: 10.1068/p7581.

26. Palmer SE, Schloss KB, Sammartino J. Visual aesthetics and human preference. Annu Rev Psychol. 2013; 64(1):77-107; doi: 10.1146/annurev-psych-120710-10 0504 .

27. Weichselbaum H, Leder H, Ansorge U. Implicit and explicit evaluation of visual symmetry as a function of art expertise. i-Perception. 2018;9(2):1-24; doi: 10.1177/ 2041669518761464.

28. Thomas JR, Nelson JK, Silverman SJ. Research methods in physical activity. Champaign: Human Kinetics; 2015.

29. Enoka RM. Neuromechanics of human movement. Champaign: Human Kinetics; 2015.

30. Rein R, Button C, Davids K, Summers J. Cluster analysis of movement patterns in multiarticular actions: a tutorial. Motor Control. 2010;14(2):211-239; doi: 10.1123/mcj.14.2.211. 